\section{La machinerie cellulaire de dégradation des protéines de l'hôte participe à la libération du génome des poxvirus}

Jason Mercer ${ }^{1}$, Pierre-Yves Lozach ${ }^{2}$
${ }^{1}$ Institut de biochimie, ETH Zürich, Schafmattstrasse 18 , 8093 Zürich, Suisse ;

${ }^{2}$ INRS-Institut Armand-Frappier, 531 boulevard des Prairies, Laval H7V 1B7, Québec, Canada.

jason.mercer@bc.biol.ethz.ch

pierre-yves.lozach@iaf.inrs.ca
Les Poxviridae, des virus hors normes Les Poxviridae sont des virus hors normes: virions visibles en microscopie optique, génome ADN de plusieurs centaines de paires de bases pouvant coder pour plus de 200 protéines, etc. [1]. Le représentant des poxvirus le plus tristement célèbre, le virus de la variole, fût à l'origine des épidémies virales les plus dévastatrices que l'humanité ait connues. Bien que la variole ait été éradiquée en 1977 suite à une campagne de vaccination internationale massive [2], l'émergence de poxvirus zoonotiques et la menace du bioterrorisme continuent de soutenir les recherches menées sur les Poxviridae.

Parmi les poxvirus, le virus de la vaccine (VVAC) est le mieux caractérisé à ce jour [3]. Son utilisation comme vaccin contre le virus de la variole et sa forte ressemblance avec ce dernier y sont pour beaucoup. Mais au-delà, les travaux sur le VVAC ont été à l'origine d'avancées majeures dans notre compréhension plus générale des virus. II est le premier virus animal à avoir été purifié, visualisé, et analysé chimiquement. Les virions arborent une structure d'une grande complexité. Ils sont enveloppés par une à deux bicouches lipidiques et comportent plus de 80 protéines. La transmission virale s'effectue via les virions à membrane unique, facilement reconnaissables en microscopie électronique à leur forme de brique (Figure IA). Chaque virion est constitué d'un core central, formé de plus de 40 protéines virales, et de deux corps latéraux adjacents (Figure lA). Alors que ces deux corps n'ont aucune fonction connue, le core protéique contient I'ADN viral et joue le rôle de capside en protégeant l'information génétique du virus. De par sa fonction protectrice, le core a une structure extrêmement stable et résiste à des stress externes intenses, comme la chaleur et l'aridité.

Le core protéique joue un rôle clé dans les étapes précoces de l'infection par le VVAC. Après leur internalisation dans la cellule hôte par macropinocytose, les virions fusionnent avec les macropinosomes et libèrent le core dans le cytosol [4]. Par un processus non encore élucidé, le core se met d'abord à grossir puis, avant de disparaître, plusieurs gènes précoces du virus s'expriment [5]. À la fin de cette séquence, I'ADN viral est libéré et débute son amplification dans le cytosol, lieu exclusif de la réplication des poxvirus. La cellule est alors infectée.

\section{Criblage à haut débit d'ARN interférents}

Comme tout virus, le VVAC est un parasite intracellulaire obligatoire qui dépend étroitement des fonctions cellulaires de son hôte pour assurer son cycle infectieux. Lors de l'étude approfondie du cycle du VVAC, notre laboratoire vient d'identifier plusieurs dizaines de facteurs cellulaires importants pour ce virus [6].
Notre travail repose sur l'extinction sélective de 7000 gènes humains au moyen de petits ARN interférents (ARNi). Pour mesurer l'effet de l'extinction de ces gènes sur l'infection par le VVAC, nous avons eu recours à des procédures complètement automatisées, aussi bien pour la transfection, l'infection, l'imagerie en microscopie à fluorescence des cellules infectées que pour l'analyse bio-informatique des données générées (Figure 1B). Cette démarche a conduit à l'identification de 188 facteurs cellulaires impliqués dans les étapes précoces de l'infection par le VVAC [6]. L'analyse des possibles interactions fonctionnelles et physiques entre ces candidats a mis en évidence plus d'une douzaine de fonctions cellulaires, hautement interconnectées, qui sont importantes pour le cycle du virus.

\section{Recycler les déchets peut nuire} à la santé

Nous avons ensuite concentré notre travail sur des groupes fonctionnels de protéines liées à la dégradation et au recyclage des protéines cellulaires: le système d'ubiquitination et le protéasome. L'ubiquitine peut être utilisée par la cellule pour marquer les protéines qui doivent être dirigées vers le protéasome pour être dégradées. Notre choix s'est arrêté à ces deux fonctions cellulaires car elles ont déjà été montrées comme nécessaires à la réplication de I'ADN du VVAC $[7,8]$. L'enjeu 


\section{A Virus de la vaccine (VVAC)}
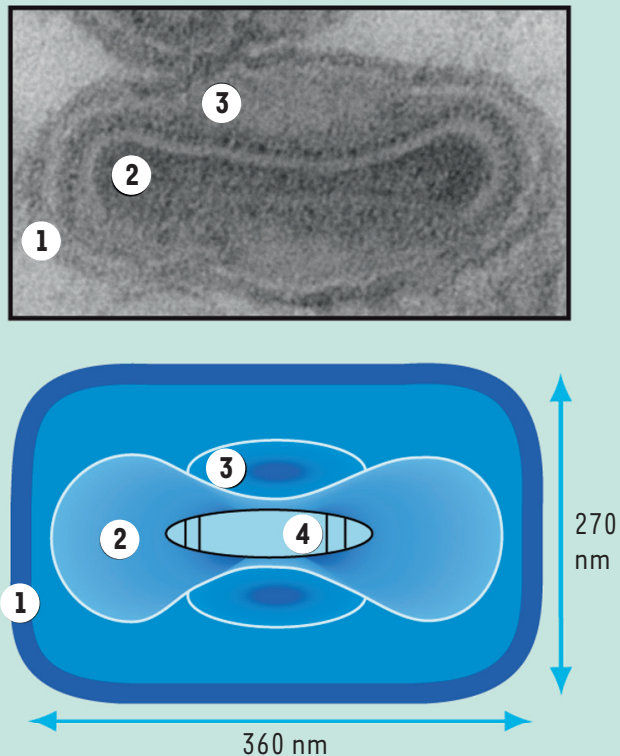

1 Membrane

3 Corps latéral

2 Core

4 Génome ADN

\section{B Criblage haut-débit d'ARNi : extinction} de 7000 gènes humains avant infection par le VVAC

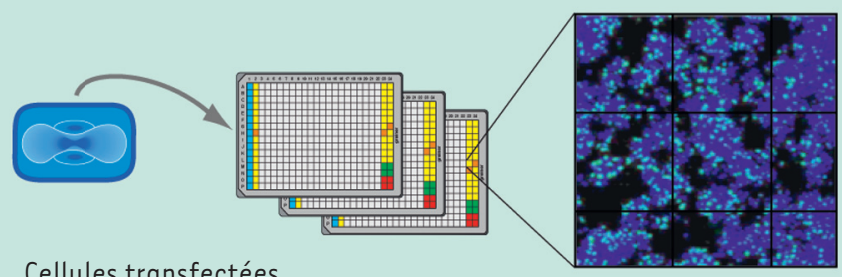

Cellules transfectées par les ARNi

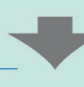

- Noyaux

Virus

Analyse bio-informatique et compilation des données

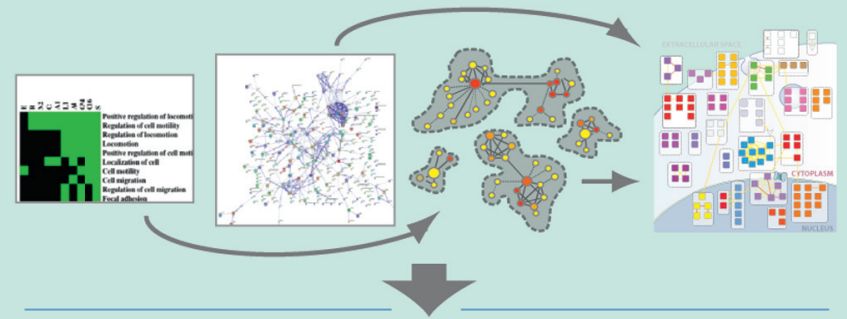

Analyse détaillée, ex. protéasome et ubiquitination
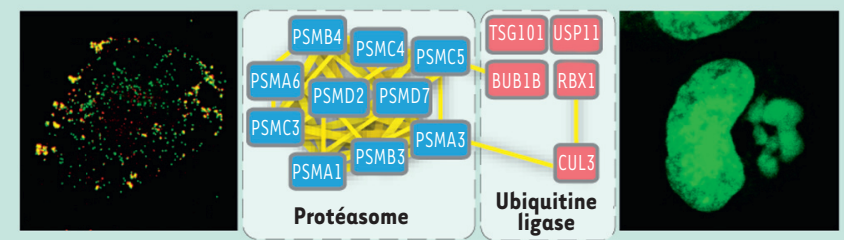

Désassemblage du core viral

C Désassemblage du core viral et réplication du génome du VVAC

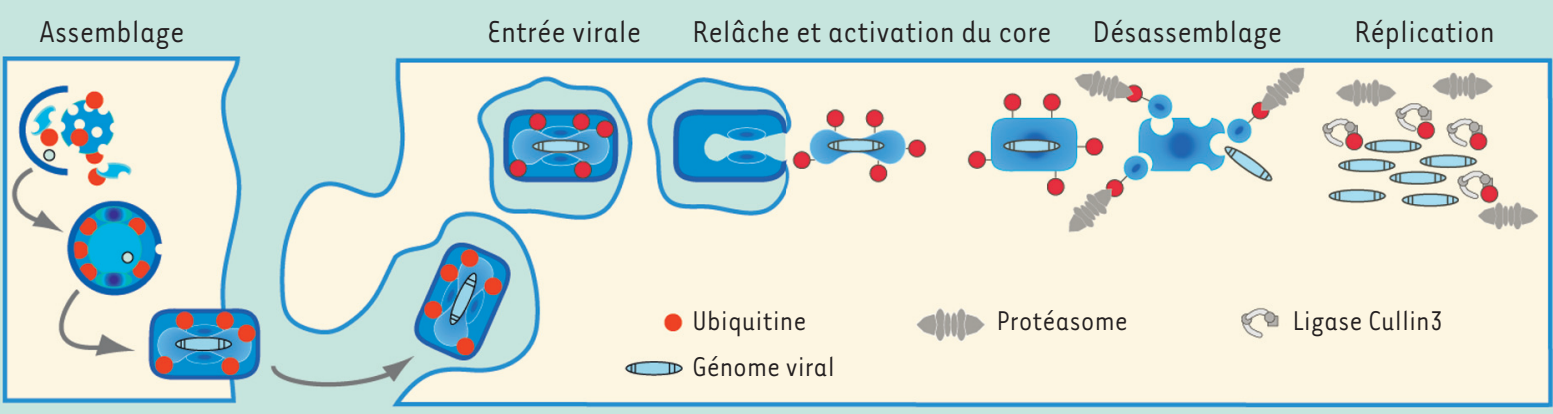

Figure 1. Rôle de l'ubiquitination et du protéasome dans le cycle du virus de la vaccine. A. Les virions matures du virus de la vaccine (VVAC) comportent plus de 80 protéines virales. En microscopie électronique, les virions ne possédant qu'une seule membrane lipidique (1) apparaissent sous forme de brique contenant un core protéique central (2) flanqué de deux corps latéraux (3). L’ADN viral (4) se trouve protégé dans le core central. Photographie de microscopie électronique reproduite avec la permission de C.K.E. Bleck (Biozentrum, université de Bâle, Suisse). B. Représentation schématique du criblage à haut-débit de petits ARN interférents ( $A R N i)$ contre 7000 gènes humains. Cette procédure a été employée pour identifier des facteurs et des fonctions de la cellule hôte importants pour le VVAC. La procédure est entièrement automatisée, de la transfection des cellules par les ARNi, en passant par l'infection par le VVAC, jusqu'à l'analyse bio-informatique des images obtenues en microscopie à fluorescence. L'analyse des interactions fonctionnelles et physiques entre les hits identifiés a permis d'établir un réseau de fonctions cellulaires étroitement interconnectées important pour le VVAC, dont celles d'ubiquitination et du protéasome. C. Modèle présentant l'implication de l'ubiquitine et du protéasome dans les différentes étapes du cycle du VVAC. PSMB : proteasome subunit ; TSG101 : tumor susceptibility gene 101 ; USP11 : ubiquitin specific protease 11 ; BUBIB : budding uninhibited by benzimidazole 1 homolog beta. 
d'une telle approche était d'asseoir la solidité des résultats issus de notre criblage en validant ces deux exemples déjà documentés dans le cas du VVAC. Notre étude a ainsi permis d'identifier les premiers facteurs cellulaires appartenant à ces groupes fonctionnels qui sont importants pour l'amplification du génome viral: Cullin3 et Rbxl (ring-box protein 1). Ces deux protéines font partie du complexe ubiquitine ligase $B R C \varepsilon 3$, un complexe responsable de l'ubiquitination des protéines devant être recyclées vers la voie du protéasome. Lorsque nous avons inactivé ce complexe, en éteignant l'expression de Cullin3 et Rbxl au moyen d'ARNi, I'ADN provenant des virions entrants n'était plus répliqué, alors que la libération du génome viral s'effectuait normalement.

Plus surprenant, nos résultats ont révélé pour la première fois que l'ubiquitination et le protéasome sont en plus essentiels au désassemblage du core protéique dans les cellules nouvellement infectées [6]. En effet, bien qu'ayant une structure très stable, le core est pourtant rapidement et efficacement désassemblé à la fin du processus d'entrée du VVAC. Nous avons pu ainsi confirmer en microscopie confocale que divers inhibiteurs de l'activité du protéasome bloquent le désassemblage du core dans lequel I'ADN viral demeure séquestré. Nos résultats confortent l'idée selon laquelle le protéasome aide à la libération du génome du virus en dégradant les protéines virales du core. $\varepsilon$ n revanche, l'emploi d'inhibiteurs de l'ubiquitination n'a eu aucun impact sur le désassemblage des cores nouvellement internalisés dans la cellule. Des marquages immunologiques ciblant différentes chaînes d'ubiquitine nous ont permis de mettre en évidence que l'ubiquitination des protéines du core avait lieu lors de la production des virions, et non lors de l'entrée du virus dans les nouvelles cellules hôtes. Cette observation est en accord avec une étude montrant que l'ubiquitine est un élément constitutif des virions [9].

\section{Conclusions et perspectives}

Notre travail est une illustration des avancées en virologie rendues possibles par les criblages à haut débit à base d'ARNi. En plus des nombreuses protéines et fonctions cellulaires identifiées, il ressort de notre étude que l'ubiquitination et le protéasome interviennent à de multiples étapes du cycle du VVAC (Figure IC). Aucun des facteurs cellulaires qui gouvernent ces mécanismes n'avait été rapporté avant notre étude [5]. De façon générale, nos résultats révèlent comment le VVAC déjoue la machinerie protéolytique de recyclage des protéines pour l'utiliser à son avantage lors de son cycle infectieux.

Bien que le protéasome ait été associé à de nombreuses infections virales [10], notre travail est la première démonstration de sa participation au désassemblage d'une capside virale ou d'une structure équivalente, comme le core du VVAC. De nombreux points restent cependant à élucider. Par quel mécanisme le core protéique est-il ubiquitiné lors de l'assemblage des virions? L'ubiquitination sert-elle de signal pour la formation des cores viraux? Comment les cores des nouveaux virions échappent-ils à la dégradation par le protéasome? Les facteurs et groupes de fonctions cellulaires identifiés ici sont autant de possibilités d'investigation pour nous permettre d'améliorer notre compréhension des mécanismes cellulaires associés au cycle infectieux des poxvirus. Une meilleure connaissance de la biologie cellulaire de ces virus devrait aider au développement de nouvelles stratégies préventives et thérapeutiques, notamment contre des poxvirus émergents, potentiellement dangereux pour l'homme. $\diamond$

Cellular protein disposal system assists poxvirus genome uncoating

\section{LIENS D'INTÉRÊT}

Les auteurs déclarent n'avoir aucun lien d'intérêt concernant les données publiées dans cet article.

\section{RÉFÉRENCES}

1. Damon IK. Poxviruses. In: Knipe DM, Howley PM, eds. Fields Virology. Philadelphia: Lippincott-Raven, 2007 2947 p.

2. Fenner F, Henderson DA, Arita I, et al. Smallpox and its eradication. Geneva: World health organization, 1988.

3. Moss B. Poxviridae: The viruses and their replication. In: Howley DMKaPM, ed. Fields Virology. Philadelphia: Lippincott-Raven, 2007 : 2906-45.

4. Mercer J, Helenius A. Vaccinia virus uses macropinocytosis and apoptotic mimicry to enter host cells. Science 2008 ; 320 : 531-5.

5. Joklik WK. The intracellular uncoating of poxvirus DNA. li. The molecular basis of the uncoating process. J Mol Biol $1964 ; 8: 277-88$.

6. Mercer J, Snijder B, Sacher R, et al. RNAi screening reveals proteasome- and Cullin3-dependent stages in vaccinia virus infection. Cell reports $2012 ; 2$ : 1036-47.

7. Satheshkumar PS, Anton LC, Sanz P, Moss B. Inhibition of the ubiquitin-proteasome system prevents vaccinia virus DNA replication and expression of intermediate and late genes. J Virol $2009 ; 83: 2469-79$.

8. Teale A, Campbell S, Van Buuren N, et al. Orthopoxviruses require a functional ubiquitinproteasome system for productive replication. J Virol 2009 ; 83 : 2099-108.

9. Chung CS, Chen CH, Ho My, et al. Vaccinia virus proteome: identification of proteins in vaccinia virus intracellular mature virion particles. J Virol 2006 ; 80 : 2127-40.

10. Banks L, Pim D, Thomas M. Viruses and the $26 \mathrm{~S}$ proteasome: hacking into destruction. Trends Biochem Sci $2003 ; 28: 452-9$.

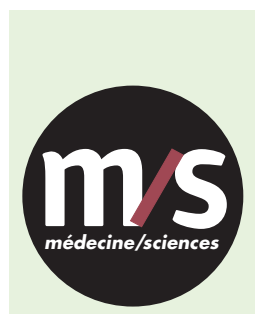

\begin{abstract}
Grâce à $m / s$, vivez en direct les progrès
Tarifs d'abonnement $m / s-2013$

des sciences biologiques et médicales
\end{abstract}

Abonnez-vous

à médecine/sciences

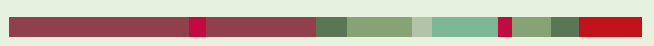

\section{Bulletin d'abonnement page 590 dans ce numéro de $\mathrm{m} / \mathrm{s}$}

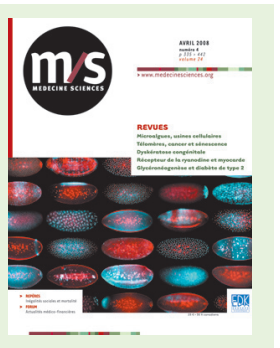

